\title{
Topical Application of Capsaicin Reduces Weight Loss Allergen Aversion and Intestinal Mucosa Inflammation in A Food Allergy Experimental Model
}

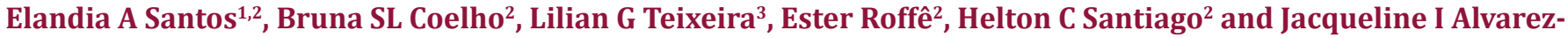 \\ Leite $^{* 2}$
}

${ }^{1}$ Departamento de Nutrição, Brazil

${ }^{2}$ Departamento de Bioquímica e Imunologia, Brazil

${ }^{3}$ Departamento de Nutrição, Brazil

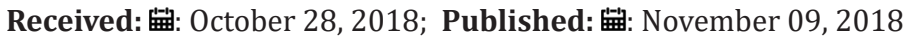

*Corresponding author: Jacqueline I Alvarez-Leite, Departamento de Bioquímica e Imunologia, Brazil

\begin{abstract}
Food allergy consists of a disease characterized by vomiting, weight loss, dehydration, and activation of inflammatory mediators in the intestinal mucosa. Capsaicin, the spicy component of pepper, is already described as an analgesic and anti-inflammatory agent. This pilot study evaluated the effect of the topical use of capsaicin on a food allergy model. Ovalbumin allergic mice (OVA) treated topically with a $100 \mathrm{mg}$ of capsaicin cream $(0.075 \%)$ or control capsaicin-free cream were challenged with OVA solution (20\%) for 7 days. Body weight, food and antigen (OVA) intakes were evaluated daily. Anti-ova IgG and IgE, neutrophil, eosinophil, and macrophage intestinal infiltration and mucus production were measured after both treatments. The results showed that allergic mice treated with topical capsaicin presented higher allergen tolerance, lower weight loss, reduced eosinophils and neutrophils intestinal infiltration and decreased mucus production compared to non-treated mice. Despite the higher intake of allergen by capsaicin-treated mice, circulating IgE and IgG antibodies concentrations were similar in both groups. We concluded that topical treatment with capsaicin attenuates the intensity of food allergy symptoms, suggesting a beneficial action in this disorder.
\end{abstract}

Keywords: Capsaicin; Food allergy; Oral tolerance

Abbreviations: TRPV1: transient receptor potential vanilloid 1 OVA: ovalbumin; MPO: myeloperoxidase EPO: eosinophil peroxidase; NAG: enzymes and n-acetylglucosaminidase TCR: T-cell receptor; TNF: Tumor Necrosis Factor IL: Interleukin; IFN: Interferon; PPAR $\gamma$ : peroxisome proliferatoractivated receptor gamma LXR: Liver X receptor

\section{Introduction}

Food allergy is an atopic disease, affecting children and adults around the world [1]. It develops when there is a failure on the induction or breakdown in oral tolerance. Due to the increasing incidence of food allergy, researches on preventive actions, including nutraceuticals agents, are growing in the literature $[2,3]$ Capsaicin (8-methyl-N-vanillyl-1-6-nonenamide) is a spicy substance found in chilies (Capsicum frutescens and Capsicum annum), with an important role in cooking. Capsaicin is a highly selective agonist for the transient receptor potential vanilloid 1 (TRPV1), a nonselective cation channel, preferably expressed by primary afferent sensory neurons of the pain pathway. This characteristic makes capsaicin potentially useful on the treatment of chronic pain [4-7]. Capsaicin creams for topical application have been commercialized to treat some inflammatory disorders. The efficacy of such an administration via has been demonstrated in studies of pain relief related to arthritis, postoperative neuralgia, diabetic neuropathy, and psoriasis $[4,8]$. Thus, we aimed to evaluate the effect of the topical use of capsaicin in a model of food allergy chronic l characterized by a ow-grade mucosa intestinal inflammation.

\section{Material and Methods}

This study was approved bt the Ethical Committee for Animal Studies of Federal University of Minas Gerais (protocol CEUA/UFMG 78/2012). Female Balb-C mice aged 5 to 8 weeks with sanitary status SPF (Specific Pathogen Free) were obtained from the animal 
facility of the Federal University of Minas Gerais, Brazil. The animals were kept in a controlled environment with 12 hours of light and dark cycle and temperature about $28^{\circ} \mathrm{C}\left( \pm 2{ }^{\circ} \mathrm{C}\right)$, with free access to food and liquids. During the challenge period (allergen intake), animals were kept in individual cages for better control of allergen intake. Food allergy was induced according to the model described by Saldanha et al. [9]. Briefly, a solution containing $10 \mu \mathrm{g}$ of ovalbumin (OVA) 98\%, $1 \mathrm{mg}$ of adjuvant (aluminum hydroxide) and $0.2 \mathrm{ml}$ of $0.9 \%$ saline solution was administrated subcutaneously on the back of the animal. After 14 days, a sensitization boost containing $10 \mu \mathrm{g}$ of OVA without adjuvant was administered. The experimental period started 7 days after the sensitization boost, by replacing water for an OVA solution (20\%). Animals were divided into the ALE group, treated topically with base cream (without capsaicin) and ALE CAPS group, which received the same base cream containing $0.075 \%$ capsaicin (v/v). Mice, without allergy induction or topic treatment were used as healthy controls.

Mice had part of their back sshaved for the daily application of $100 \mathrm{mg}$ of the respective creams in an area of approximately 1 $\mathrm{cm}^{2}$ days during 7 days, according to a previous protocol [10]. Food and liquid intakes were measured daily by the difference of the quantity offered and the rest. All animals were euthanized on the 8th experimental day and blood, and small intestine samples were withdrawn for further analyses. Blood were used for the quantification of anti OVA IgE and IgG1 antibodies concentration by ELISA kits to confirm allergy induction. The evaluation of the cellular infiltrate of the small intestine was made by the myeloperoxidase (MPO), eosinophil peroxidase (EPO) enzymes and n-acetylglucosaminidase (NAG) activities, as indirect measure of neutrophils, eosinophils, and macrophages, respectively. Histopathological analysis of the goblet cells producing mucus was performed in the small intestine by the periodic acid from Schiff
(P.A.S) stain using the Image J. Software (NIH, USA). PAS positive pixels were selected for the calculation of the total area mucusproducing goblet cells. The result was expressed as \% of P.A.S positive area of intestinal mucosa/field. The data were evaluated for normal distribution by the Kolmogorov-Smirnov test. Parametric and non-parametric data were submitted to Student's t-Test and Mann Whitney test, respectivelly. The results were expressed as the mean and standard error. Statistical analyzes were performed using GraphPad Prism 7.0® software (San Diego, CA, USA). The level of significance was set at $\mathrm{p}<0.05$.

\section{Results}

The results of blood concentration showed that anti OVA IgE and IgG1 antibodies levels were compatible with the presence of OVA allergy. However, no difference was detected between ALE and ALE CAPS groups considering IgE or IgG1 concentrations (Figure 1). Although IgE and IgG1 levels were similar in both groups, the OVA solution intake, in turn, was higher in animals treated with topical capsaicin, showing a higher tolerance to the allergen (Figure 1). Food intake was reduced compared to healthy mice, but similar in allergic groups confirming a similar caloric intake between ALE and ALE CAPS groups. Nonetheless, ALE CAPS mice showed a better body weight evolution, with a weight loss lower than in ALE group during the experimental days (Figure 1). When the intestinal inflammatory infiltrates were analyzed, it was noted that the ALE CAPS group showed lower neutrophil and eosinophil infiltration, as suggested by the MPO and EPO activity, respectively (Figure 2). No difference was seen when the macrophage infiltration was compared $(p=0.064)$. ALE group exhibited hypersecretion of mucus by goblet cells. However, a smaller mucus production was observed in allergic animals treated with capsaicin when compared to allergic animals (Figure 3).

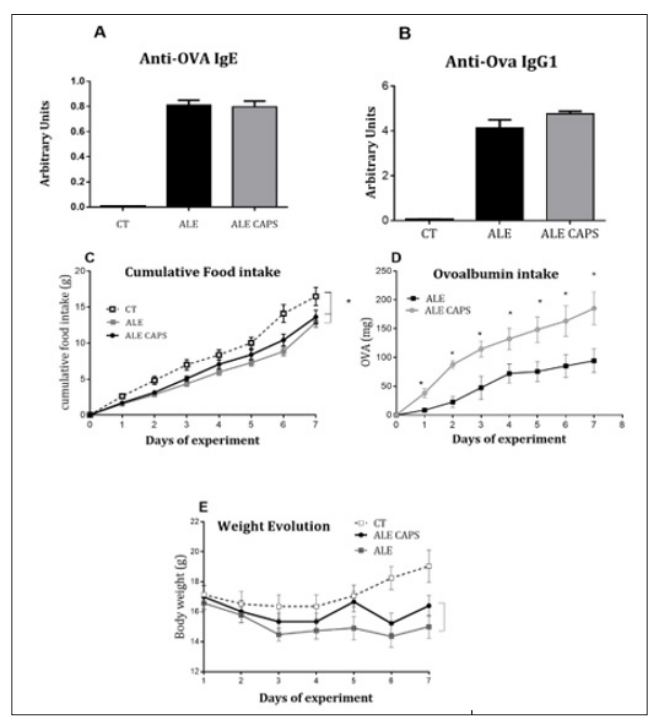

Figure 1: Evaluation of blood levels of $\operatorname{IgE}(\mathrm{A})$ and $\operatorname{IgG1}(\mathrm{B})$, cumulative food intake (C), ovalbumin intake (D) and weight evolution (E) in ovalbumin allergic mice topically treated with capsaicin-free cream (ALE), 0.075\% capsaicin cream (ALE CAPS) or in mice without allergy or capsaicin treatment (CT). The results were expressed as mean and SD. SD. N=7/group. * Statistically significant $(\mathrm{p}<0.05)$. 
A

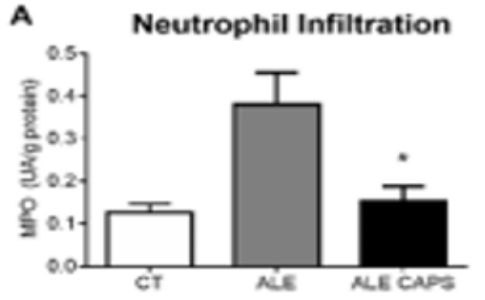

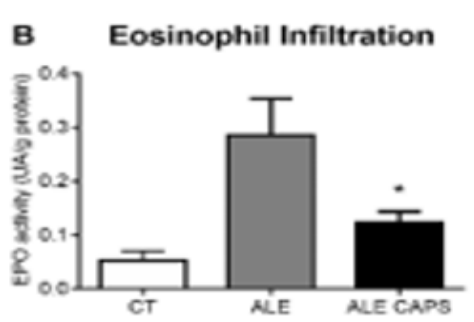

Figure 2: Indirect evaluation of neutrophil (A), eosinophil (B) and macrophage (C) infiltration assessed by the activity of the enzymes myeloperoxidase (MPO), eosinophil peroxidase (EPO) and n- acetylglucosaminidase (NAG), respectively in the small intestine of ovalbumin allergic mice topically treated with capsaicin-free cream (ALE), 0.075\% capsaicin cream (ALE CAPS) in mice without allergy or capsaicin treatment (CT). Bars represent the means and vertical lines the standard error of 7 animals/group. ${ }^{*} \mathrm{p}<0.05$ compared to ALE group.

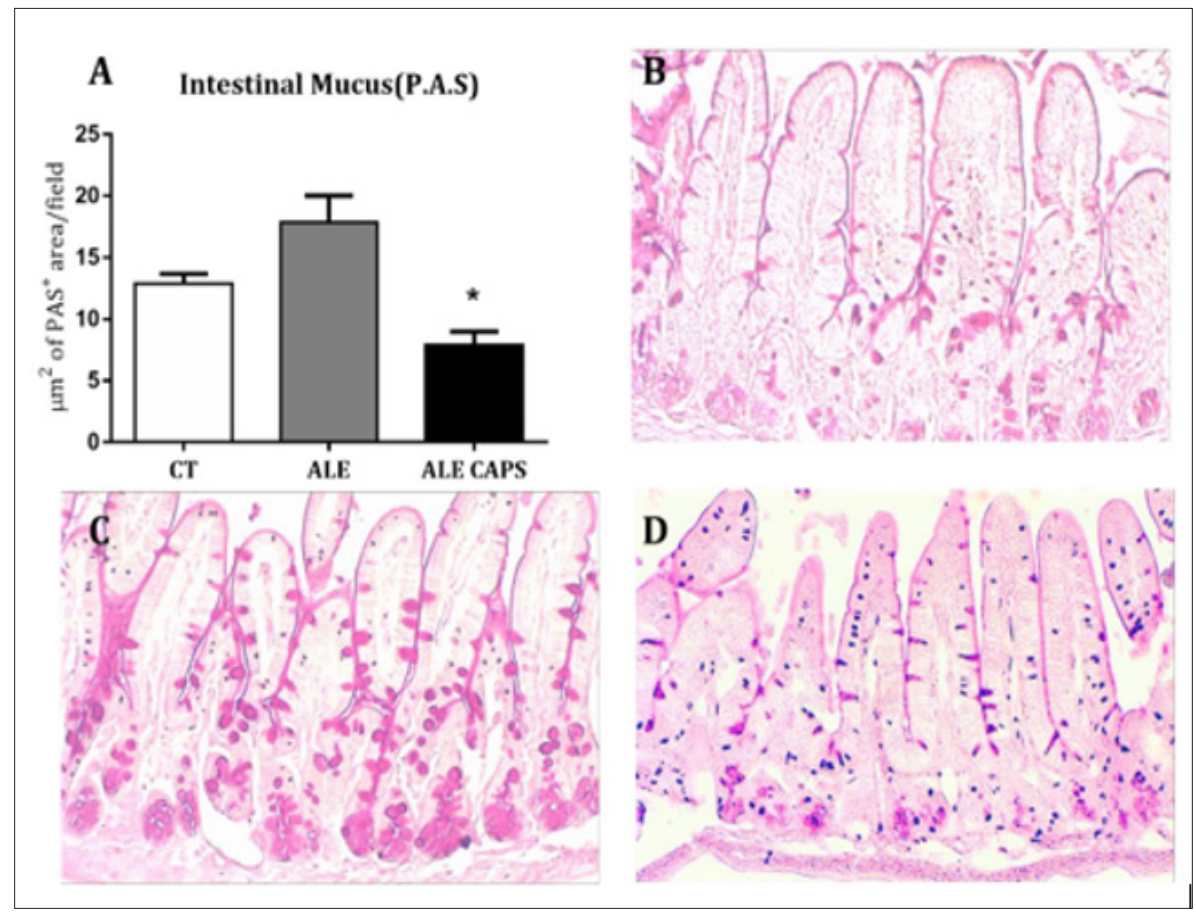

Figure 3: Evaluation of the neutral mucus produced by the goblet cells of the small intestine. (A) Quantification of the mucus represented as the intestinal mucosa PAS-positive area $\left(\mu \mathrm{m}^{2}\right)$ by field. Bars represent the media and standard error of 4 animals per group. ${ }^{*} \mathrm{p}<0.05$ compared to ALE group. Histological analysis (P.A.S) of intestinal mucosa of control mice (B) or ovalbumin allergic mice topically treated with (C) capsaicin-free cream (ALE) or (D) $0.075 \%$ capsaicin cream (ALE CAPS).

\section{Discussion}

Our results suggest a beneficial effect of the topical application of capsaicin in reducing the manifestations of food allergy. Due to its lipophilic character, capsaicin can be absorbed from the skin, with a half-life of about 24 hours [4]. This route of administration has already been used in commercial products to help relieve a several types of minor neuralgia related to disorders such as rheumatoid arthritis or muscle sprains and strains $[11,12]$.

Two of the major signs of food allergy are weight loss and allergen aversion [13,14]. Our results show that capsaicin can reduce both since the ALE CAPS group presented higher intake of OVA solution and lower weight loss compared to ALE mice. The improvement in allergy in the capsaicin- treated group was also demonstrated by the lower recruitment of neutrophils and eosinophils to the small intestine. Neutrophils are the first cells to be recruited to the site of inflammation, being installed in the lamina propria and intestinal crypts, releasing the enzyme myeloperoxidase (MPO) among other components [15]. Eosinophils are fundamental cells in the allergic process, serving as "immunological effector sentinels", with rapid action in inflammatory processes $[13,16]$. In allergic diseases, eosinophils are activated and attracted to the intestinal mucosa, releasing the degranulation products such as toxins and free radicals, 
which can trigger intestinal injury. The release of leukotrienes and cytokines by eosinophils amplifies the inflammatory response, recruiting and activating more eosinophils. The mucosa eosinophil infiltration in others intestinal disorder such as inflammatory bowel diseases is associated with unfavorable conditions such as malabsorption, weight loss and changes in mucosal architecture with decreased crypts in the colon and villous flattening [16-18].

These results suggest that topical capsaicin is acting as an anti-inflammatory agent creating a less inflamed intestinal environment. Another important feature of food allergy is the increased production of mucus by goblet cells [13]. It is known that in the late phase of food allergy, eosinophils release lipid mediators such as prostaglandin D2, which increase mucus production by intestinal goblet cells [13]. Our data suggested that the lower production of mucus in the ALE CAPS group could be the result of the lower eosinophil infiltration in this group and its lower release of lipid mediators. Our data agree with the hypothesis that topical capsaicin would act mainly in the late phase of food allergy leading to the lower infiltration of inflammatory cells. Although the antiinflammatory action of capsaicin has been described in several in vitro studies, the mechanisms are not fully understood $[4,19,20]$. One of the possible mechanisms is related to the action of capsaicin as a TRPV1 ligand [21].

It has been described that for the complete activation of the TRPV1 vanilloid channel, about $10 \mu \mathrm{M}$ of capsaicin is required [22-26]. When activated, TRPV1 channels are associated with thermal hyperalgesia in the skin, visceral organs and oral cavity [21]. However, repeated exposures to capsaicin, as occurred in the present study, cause effects of analgesia due to the nociceptor desensitization or a calcium-dependent extracellular reduction and TRPV1 responsiveness [4,22,23,26-29]. This inhibition, in turn, regulates T-cell receptor (TCR) -mediated T cell activation with the production of anti-inflammatory cytokines through the suppression of TNF- $\alpha$, IL-2 and IFN- $\alpha$ [30]. Capsaicin appears to be also a direct agonist of the peroxisome proliferator-activated receptor gamma (PPAR $\gamma$ ) that can stimulate the liver X receptor (LXR) expression in an anti-inflammatory pathway, independent of TRPV1 activation $[20,21]$. These results are in agreement with those of Lee, Shin et al. who observed lower expression of proinflammatory cytokines in obese mice topically treated with capsaicin, reinforcing the regulatory role of capsaicin on inflammation [10]. Although all parameters demonstrated improvement of food allergy symptoms with topical use of capsaicin, IgE and IgG1 concentrations were similar between groups. However, due to the 3 and 23-day halflives of these immunoglobulins, respectively [31], the treatment time (7 days) may not have been sufficient to detect significant changes in their plasma levels. Alternatively, if capsaicin somehow interferes with IgE binding with its Fc $\varepsilon$ RI receptor rather than reducing IgE production, no difference in the circulating levels of that immunoglobulin would be detected.

\section{Conclusion}

Our data suggest that the topical application of capsaicin is associated to positive effects in food allergy related to reduction of major manifestations, such as allergen aversion and body weight loss.

\section{Acknowledgment}

This study was supported by Fundação de Amparo à Pesquisa do Estado de Minas Gerais (FAPEMIG), Coordenação de Aperfeiçoamento de Pessoal de Nível Superior (CAPES), Conselho Nacional de Desenvolvimento Científico e Tecnológico (CNPq) and by Pro-reitoria de Pesquisa (PRPq) of Universidade Federal Minas Gerais. The authors thank Maria Helena Alves de Oliveira and for the support in animal facility.

\section{References}

1. Camps Bossacoma M, Pérez-Cano FJ, Franch À, Untersmayr E, Castell M (2017) Effect of a cocoa diet on the small intestine and gut- associated lymphoid tissue composition in an oral sensitization model in rats. The Journal of Nutritional Biochemistry 42: 82-193.

2. Bauer RN, Manohar M, Singh AM, Jay DC, Nadeau KC (2015) The future of biologics: Applications for food allergy. Journal of Allergy and Clinical Immunology 135(2): 312-323.

3. Noval Rivas M, Burton OT, Wise P, Charbonnier LM, Georgiev P, et al. (2015) Regulatory $\mathrm{T}$ Cell Reprogramming toward a Th2-Cell-like Lineage Impairs Oral Tolerance and Promotes Food Allergy. Immunity 42(3): 512-523.

4. O Neill J, Brock C, Olesen AE, Andresen T, Nilsson M, et al. (2012) Unravelling the Mystery of Capsaicin: A Tool to Understand and Treat Pain. Pharmacological Reviews 64(4): 939-971.

5. Frias B, A Merighi (2016) Capsaicin, Nociception and Pain. Molecules 21(6): 797.

6. Yang F, J Zheng, (2017) Understand spiciness: mechanism of TRPV1 channel activation by capsaicin. Protein Cell 8(3): 169-177.

7. Talbot S, SL Foster, CJ Woolf (2016) Neuroimmunity: Physiology and Pathology. Annu Rev Immunol 34: 421-447.

8. Henrich F, Magerl W, Klein T, Greffrath W, Treede RD (2015) Capsaicinsensitive C- and A-fibre nociceptors control long-term potentiation-like pain amplification in humans. Brain 138(9): 2505-2520.

9. Saldanha JC, Gargiulo DL, Silva SS, Carmo Pinto FH, Andrade MC, et al. (2004) A model of chronic IgE-mediated food allergy in ovalbuminsensitized mice. Brazilian Journal of Medical and Biological Research 37(6): 809- 816.

10. Lee GR, Shin MK, Yoon DJ, Kim AR, Yu R, et al. (2013) Topical application of capsaicin reduces visceral adipose fat by affecting adipokine levels in high-fat diet-induced obese mice. Obesity (Silver Spring) 21(1): 115122 .

11. (2018) Drugs and Supplements - Capsaicin (Topical Route). Mayo Clinic.

12. Kongkiat K, L Chakraphong, M Pongsatorn (2013) 0.025\% Capsaicin Gel for the Treatment of Painful Diabetic Neuropathy: A Randomized, Double-Blind, Crossover, Placebo-Controlled Trial. Pain Practice 13(6): 497-503.

13. Bhalla PL, MB Singh (2008) Biotechnology-based allergy diagnosis and vaccination. Trends in Biotechnology 26(3): 153-161.

14. Valenta R, Heidrun Hochwallner, Birgit Linhart, Sandra Pahr (2015) Food Allergies: The Basics. Gastroenterology 148(6): 1120- 1131.

15. Naito Y, T Takagi, T Yoshikawa (2007) Neutrophil-Dependent Oxidative Stress in Ulcerative Colitis. Journal of Clinical Biochemistry and Nutrition 41(1): 18-26.

16. Leonel AJ, Lílian G Teixeira, Rafael Pires de Oliveira, Andrezza Santiago (2012) Antioxidative and immunomodulatory effects of tributyrin 
supplementation on experimental colitis. British Journal of Nutrition 109(8): 1396-1407.

17. Vieira EL, Leonel AJ, Sad AP, Beltrão NR, Costa TF, et al. (2012) Oral administration of sodium butyrate attenuates inflammation and mucosal lesion in experimental acute ulcerative colitis. The Journal of Nutritional Biochemistry 23(5): 430-436.

18. Woerly G, Roger N, Loiseau S, Dombrowicz D, Capron A, et al. (1999) Expression of $\mathrm{Cd} 28$ and Cd86 by Human Eosinophils and Role in the Secretion of Type 1 Cytokines (Interleukin 2 and Interferon $\gamma$ ): Inhibition by Immunoglobulin a Complexes. The Journal of Experimental Medicine 190(4): 487-496.

19. Srinivasan K (2015) Biological Activities of Red Pepper (Capsicum annuum) and Its Pungent Principle Capsaicin: A Review. Critical Reviews in Food Science and Nutrition p. 00-00.

20. Tang J, Luo K, Li Y, Chen Q Tang D, et al. (2015) Capsaicin attenuates LPSinduced inflammatory cytokine production by upregulation of LXR $\alpha$. International Immunopharmacology 28(1): 264-269.

21. Park JY, Kawada T, Han IS, Kim BS, Goto T, et al. (2004) Capsaicin inhibits the production of tumor necrosis factor $\alpha$ by LPS- stimulated murine macrophages, RAW 264.7: a PPAR $\gamma$ ligand-like action as a novel mechanism. FEBS Letters 572(1): 266-270.

22. Clapham DE (2003) TRP channels as cellular sensors. Nature 426(6966): 517-524.

23. Fernandes ES, MA Fernandes, JE Keeble (2012) The functions of TRPA1 and TRPV1: moving away from sensory nerves. British Journal of Pharmacology 166(2): 510- 521

\section{ISSN: 2574-1241}

DOI: 10.26717/BJSTR.2018.10.002023

Jacqueline I Alvarez-Leite. Biomed J Sci \& Tech Res

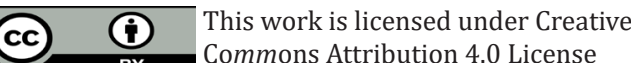

Submission Link: https://biomedres.us/submit-manuscript.php
24. Lanser BJ, Wright BL, Orgel KA, Vickery BP, Fleischer DM (2015) Current Options for the Treatment of Food Allergy. Pediatr Clin North Am 62(6): 1531-1549.

25. Elokely K, Velisetty P, Delemotte L, Palovcak E, Klein ML, et al. (2016) Understanding TRPV1 activation by ligands: Insights from the binding modes of capsaicin and resiniferatoxin. Proc Natl Acad Sci U S A 113(2): 137- 145 .

26. Zhang W, G Yu, M Zhang (2016) ARA 290 relieves pathophysiological pain by targeting TRPV1 channel: Integration between immune system and nociception. Peptides 76: 73-79.

27. Reilly CA, William J Ehlhardt, David A Jackson, Palaniappan Kulanthaivel, Abdul EM, et al. (2003) Metabolism of Capsaicin by Cytochrome P450 Produces Novel Dehydrogenated Metabolites and Decreases Cytotoxicity to Lung and Liver Cells. Chemical Research in Toxicology 16(3): 336-349.

28. Chanda S, Bashir M, Babbar S, Koganti A, Bley K, et al. (2008) In Vitro Hepatic and Skin Metabolism of Capsaicin. Drug Metabolism and Disposition 36(4): 670-675.

29. Murakami S, Sudo Y, Miyano K, Nishimura H, Matoba M, et al. (2016) Tris-hydroxymethyl-aminomethane enhances capsaicin-induced intracellular $\mathrm{Ca}(2+)$ influx through transient receptor potential V1 (TRPV1) channels. J Pharmacol Sci 130(2): 72-77.

30. Deng Y, Huang X, Wu H, Zhao M, Lu Q et al. (2016) Some like it hot: The emerging role of spicy food (capsaicin) in autoimmune diseases. Autoimmunity Reviews 15(5): 451-456.

31. Hardy RR (2013) B-Lymphocyte Development and Biology in Fundamental Immunology, WE Paul, Editor. Lippincott Williams \& Wilkins: Philadelphia p. 215-245.

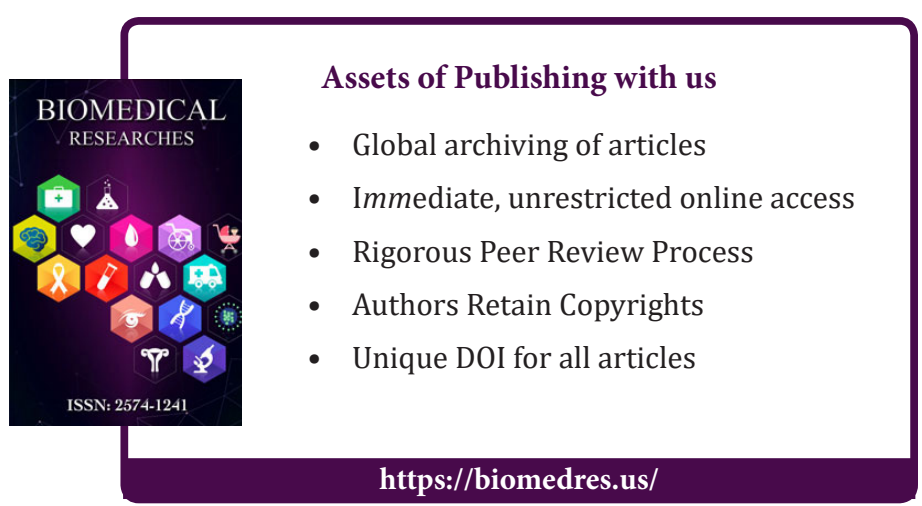

